\title{
Feasibility of endoscopic suturing to prevent adverse events and hospitalization after endoscopic submucosal dissection $\nabla$
}

\section{다 (1) $(2)$}

\author{
Authors \\ Institutions \\ 1 Division of Gastroenterology and Hepatology, University \\ of Colorado Anschutz Medical Campus, Aurora, \\ Colorado, United States \\ 2 Advanced Gastrointestinal Endoscopy, Mountain View, \\ California, United States
}

Samuel Han ${ }^{1}$, Sachin Wani ${ }^{1}$, Steven A. Edmundowicz ${ }^{1}$, Roy Soetikno², Hazem Hammad ${ }^{1}$

submitted 17.3.2020

accepted after revision $\quad 19.5 .2020$

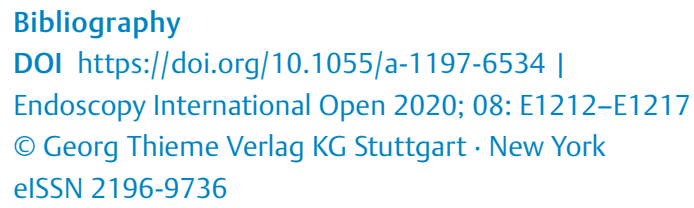

\section{ABSTRACT}

Background and study aim Endoscopic submucosal dissection (ESD) enables en bloc removal of gastrointestinal epithelial lesions but can leave a large mucosal defect, which can lead to inpatient observation and delayed bleeding or perforation. The aim of this study was to examine the safety and effectiveness of endoscopic suturing in closing ESD defects to prevent adverse events.

Patients and methods In this single-center prospective cohort study, endoscopic suturing was performed to close ESD defects in the stomach or rectum. Suturing was performed in the antegrade position starting from the edge most distal to the endoscope insertion site, moving from right to left, left to right manner before ending at the edge most proximal to the endoscope insertion site.

Results In total, 31 patients (mean age $65.6,71 \%$ male) received endoscopic suturing after gastric $(58.1 \%)$ or rectal (41.9\%) ESD. Mean lesion size was $27.4 \pm 16.2 \mathrm{~mm}$ and mean suturing time was $13.4 \pm 5.9 \mathrm{~min}$. Complete closure was achieved in all patients. Same-day discharge occurred in $58.1 \%$ of patients; the remainder were hospitalized with mean length of stay of $1 \pm 0.6$ day. There were no instances of delayed bleeding or delayed perforation $(0 \%, 95 \% \mathrm{Cl}$ : $0-$ $11.5 \%)$. No recurrences were found on surveillance endoscopy.

Conclusions Based on this small prospective study, endoscopic suturing of post-ESD defects in the stomach and rectum appears to be feasible, safe, and potentially effective in preventing bleeding or perforation. Further larger controlled studies, however, are needed to validate these findings.

\section{Introduction}

Endoscopic submucosal dissection (ESD) enables en bloc removal of lesions throughout the gastrointestinal tract by cutting through the submucosa below the lesion [1]. While offering the potential for curative resection, ESD requires specialized training and experience and remains technically challenging with a relatively high rate of adverse events (AEs) including in- traprocedural and delayed bleeding (4.5\%-15.6\%), immediate and delayed perforation (2.3\%), and stricture formation (12\%$17 \%)$ [1]. The large defects left from ESD often result in ulceration and patients are often hospitalized for precautionary measures [2].

To potentially improve healing and prevent related AEs, various methods have been described to close these post-ESD defects. The most common method entails prophylactic clipping 
with standard hemostatic clips or an over-the-scope-clip which remains challenging in large defects [3-5]. More advanced closure techniques include using clips to anchor an endoloop in a purse-string manner or hand-suturing through an overtube [6, 7]. Endoscopic suturing via the Overstitch endoscopic suturing (Apollo Endosurgery, Austin, Texas, United States) offers another potential approach to closing these defects, but has only been studied in a small retrospective series [8].

The aim of this prospective study was to examine the feasibility, safety and effectiveness of endoscopic suturing in closing ESD defects to prevent AEs such as bleeding and perforation.

\section{Patients and methods}

This single-center prospective cohort study received approval from the Colorado Multiple Institutional Review Board and the Veterans Affairs Central Institutional Review Board.

\section{Patients}

Inclusion criteria included all patients with gastric or rectal lesions resected using ESD from July, 2018 to July, 2019. Patients with gastric or rectal lesions were consecutively enrolled with endoscopic suturing performed in all study participants. Demographics, procedural details, AEs, and surveillance results were recorded. Follow-up was performed via patient phone calls and assessment of medical records. The first surveillance endoscopy was typically scheduled at 6 months.

All patients provided informed consent to the study and all procedures were performed at a tertiary academic medical center or its affiliated Veterans Affairs regional medical center by a single expert endoscopist $(\mathrm{HH})$.

\section{Endoscopic suturing technique}

ESD was performed using a standard gastroscope (Olympus, Tokyo, Japan) in either the stomach or rectum. During ESD, a Coagrasper hemostatics forceps (Olympus) and coagulation with the knife was used to treat vessels during the dissection and any visible vessles in the base of the resection defect at the end. Upon completion of ESD, the Overstitch endoscopic suturing platform was attached to a double-channel therapeutic gastroscope (Olympus) and advanced to the site of ESD. As previously described, all suturing was performed in the antegrade position starting from the edge of the defect most distal to the insertion site of the endoscope ( Video 1 ) $[9,10]$. As opposed to performing full-thickness suturing, the suture was placed through the mucosal and submucosal flap and once the initial flap was grasped, suturing was performed in a continuous fashion advancing in a right to left, left to right manner until the edge most proximal to the endoscope insertion site was grasped for a total of approximately 6 flaps based on the size of the defect ( $\triangleright$ Fig. 1). The distance between the bites is typically 1 to $2 \mathrm{~cm}$. Full-thickness bites were intentionally not taken to prevent any potential injury to the muscularis propria. The cinch was then deployed and repeat examination of the defect site was performed with the suturing platform removed ( $\vee$ Fig. 2, $>$ Fig. 3, $>$ Fig.4). The decision to admit the patient was based on the patient's level of pain and nausea post-proce-

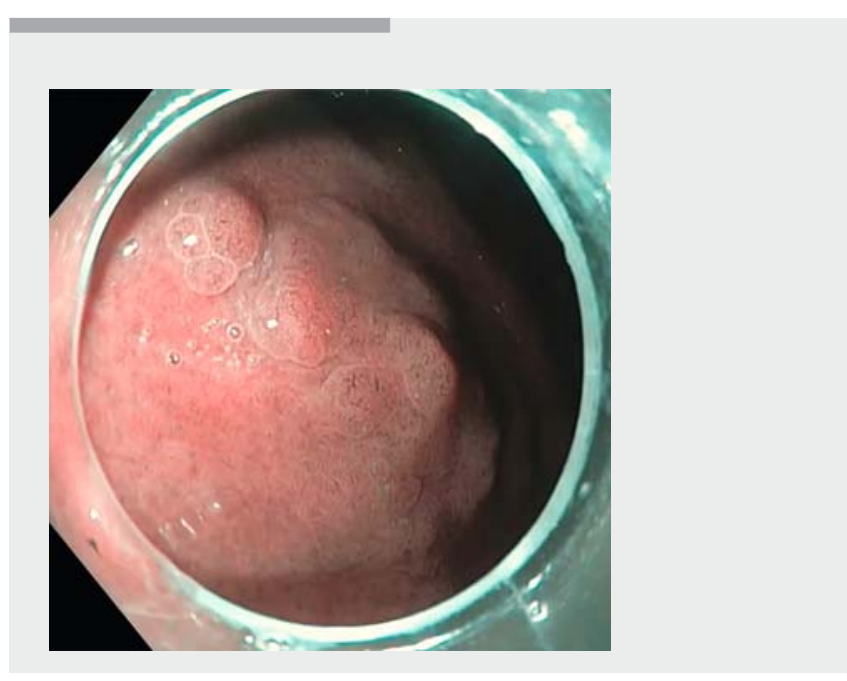

Video 1 Endoscopic suturing after gastric endoscopic submucosal dissection.

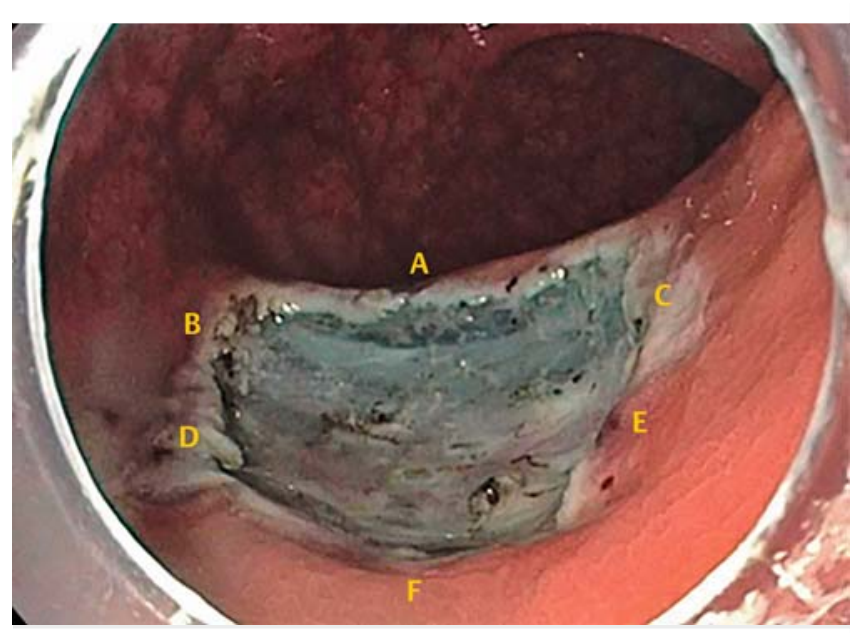

- Fig. 1 Schema for suturing defect resulting from endoscopic submucosal dissection $(A \rightarrow B \rightarrow C \rightarrow D \rightarrow E \rightarrow F)$.

dure as well as the availability of transportion home. After gastric ESD, patients were placed on twice-daily proton pump inhibitor for 4 weeks. If not on antithrombotics/anticoagulation, patients were advised to avoid aspirin and non-steroidal anti-inflammatory medications for 1 week.

\section{Outcomes}

Technical success was defined as successful closure of the ESD using the aforementioned technique. In terms of AEs, intraprocedural bleeding was defined as bleeding occurring during ESD with significant bleeding defined as active bleeding requiring the use of additional tools to control (such as hemostatic forceps or epinephrine injection). Delayed bleeding was defined as overt active bleeding occurring up to 15 days post-ESD (hematemesis, melena, and/or hematochezia). Delayed perforations included perforations that were not recognized intraprocedurally and occurred up to 15 days post-ESD. Submucosal dissection time was defined as the time from initial incision to 

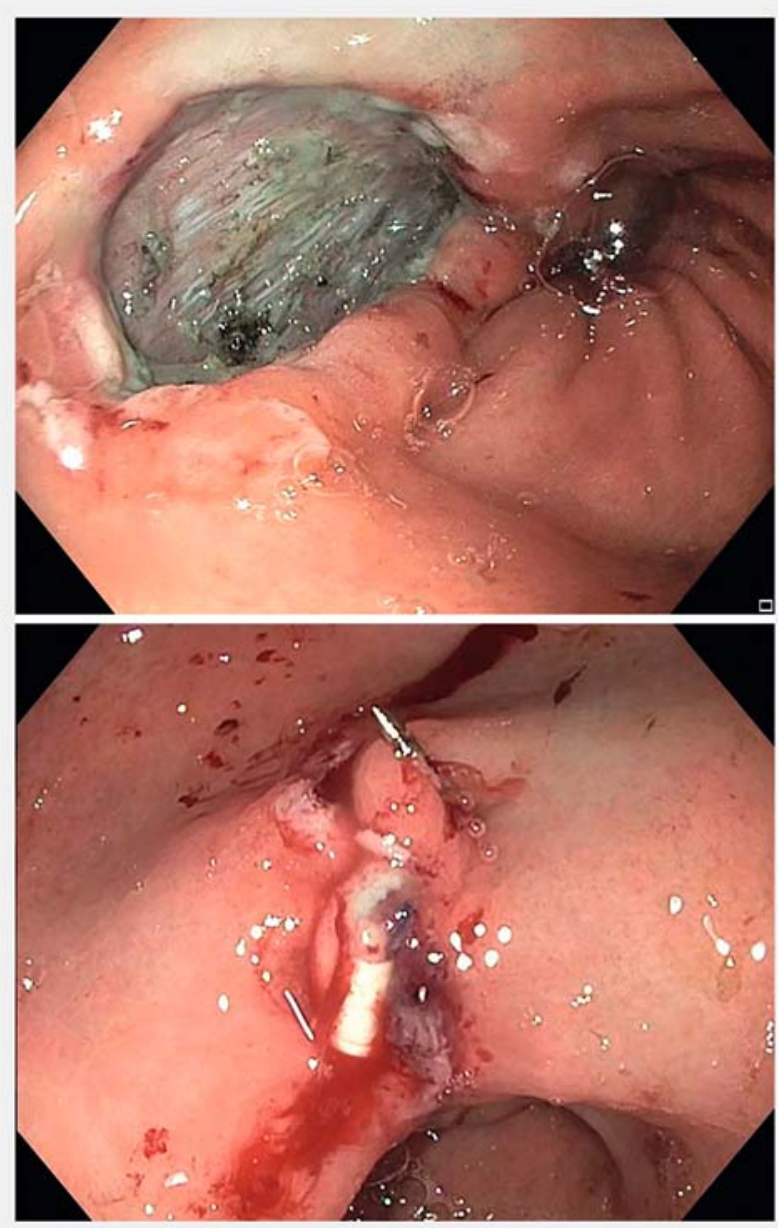

- Fig. 2 Gastric endoscopic submucosal dissection defect site before (above) and after (below) endoscopic suturing.

completion of lesion dissection. Endoscopic suturing time was defined as the time from insertion of the endoscopic suturing platform to the resection site to deployment of the cinch.

\section{Statistical analysis and sample size}

We chose a sample size of 30 patients in accordance with the upper confidence limit approach to estimate the variance of the outcome measure (delayed bleeding and perforation) [11]. Summary statistics were performed using STATA 15.1 (College Station, Texas, United States) to calculate means, standard deviations (SD) and frequencies.

\section{Results}

In total, 31 patients (mean age $65.6,71 \%$ male) treated with gastric $(58.1 \%)$ or rectal $(41.9 \%)$ ESD (mean lesion size of 27.4 $\pm 16.2 \mathrm{~mm}$, resected specimen size of $38.7 \pm 16.1 \mathrm{~mm}$ ) had post-ESD defects closed via endoscopic suturing ( $\vee$ Table $\mathbf{1})$. The most common lesion pathology was adenocarcinoma (22.6\%) followed by adenomas with high-grade dysplasia (19.4\%) and low-grade dysplasia (19.4\%). Mean dissection
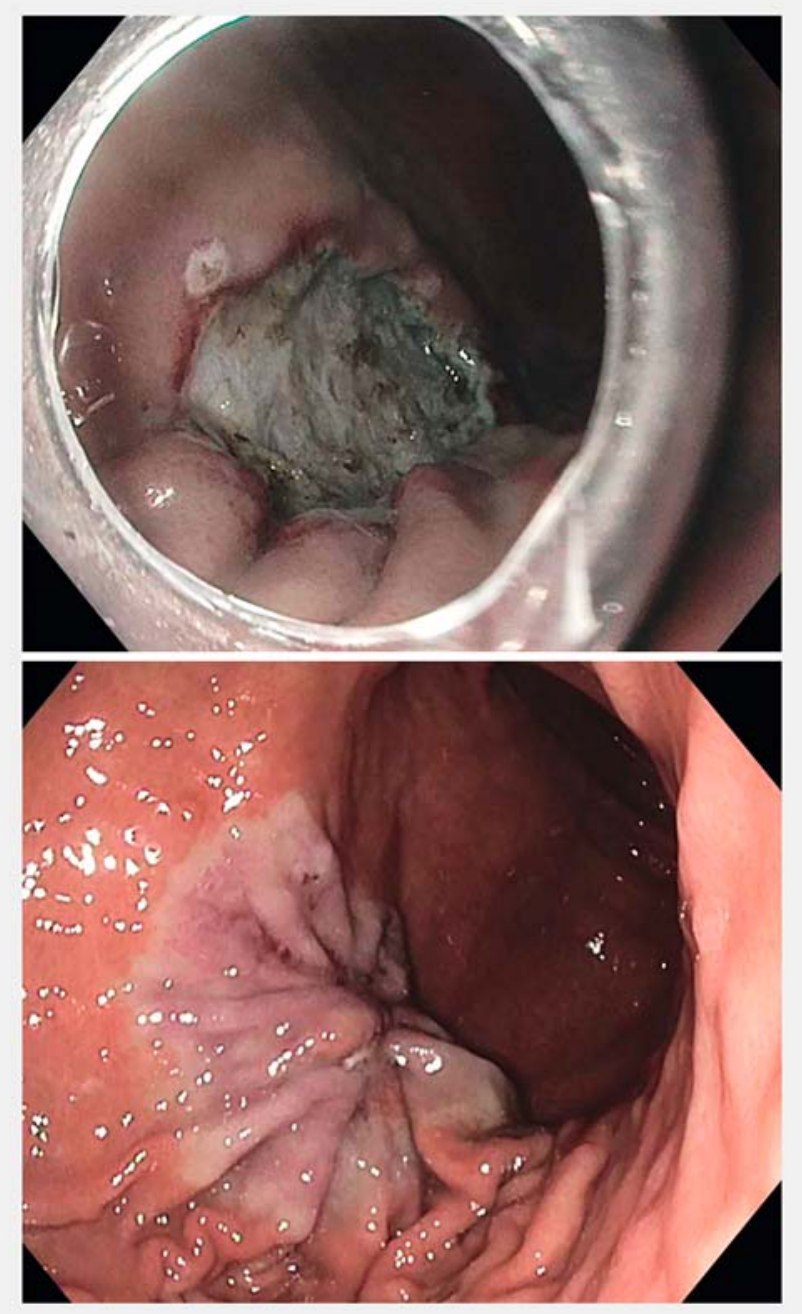

- Fig. 3 Endoscopic submucosal dissection defect site in stomach before (above) and after (below) endoscopic suturing.

time was 54.8 (SD 34.6) minutes and mean suturing time was 13.4 (SD 5.9) minutes. Two (6.5\%) patients used antiplatelet agents and three $(9.7 \%)$ patients were on anticoagulation at baseline.

Technial success was achieved in all patients. Significant intraprocedural bleeding occurred in $2(6.5 \%)$ cases (both treated with hemostatic forceps) with no instances of intraprocedural perforation ( $0 \%, 95 \% \mathrm{Cl}: 0-11.2 \%)$. Same-day discharge occurred in $58.1 \%(n=18)$ of patients with a mean length of stay of 1 (SD 0.6) day in those who required overnight admission. Two patients were unable to be discharged home the same day due to a lack of transportation. No episodes of delayed bleeding ( $0 \%, 95 \% \mathrm{Cl}: 0-11.2 \%)$ or delayed perforation (0\%, $95 \% \mathrm{Cl}: 0-11.2 \%)$ occurred.

A mean of 1.29 (SD 0.7) surveillance endoscopies have been performed in 21 patients thus far at a mean of 143.9 (SD 42.2) days. No residual adenoma/malignancy have been found and during a mean follow-up period of 292.7 (SD 170.6) days, there have been no recurrences. Furthermore, no cases of tissue inva- 


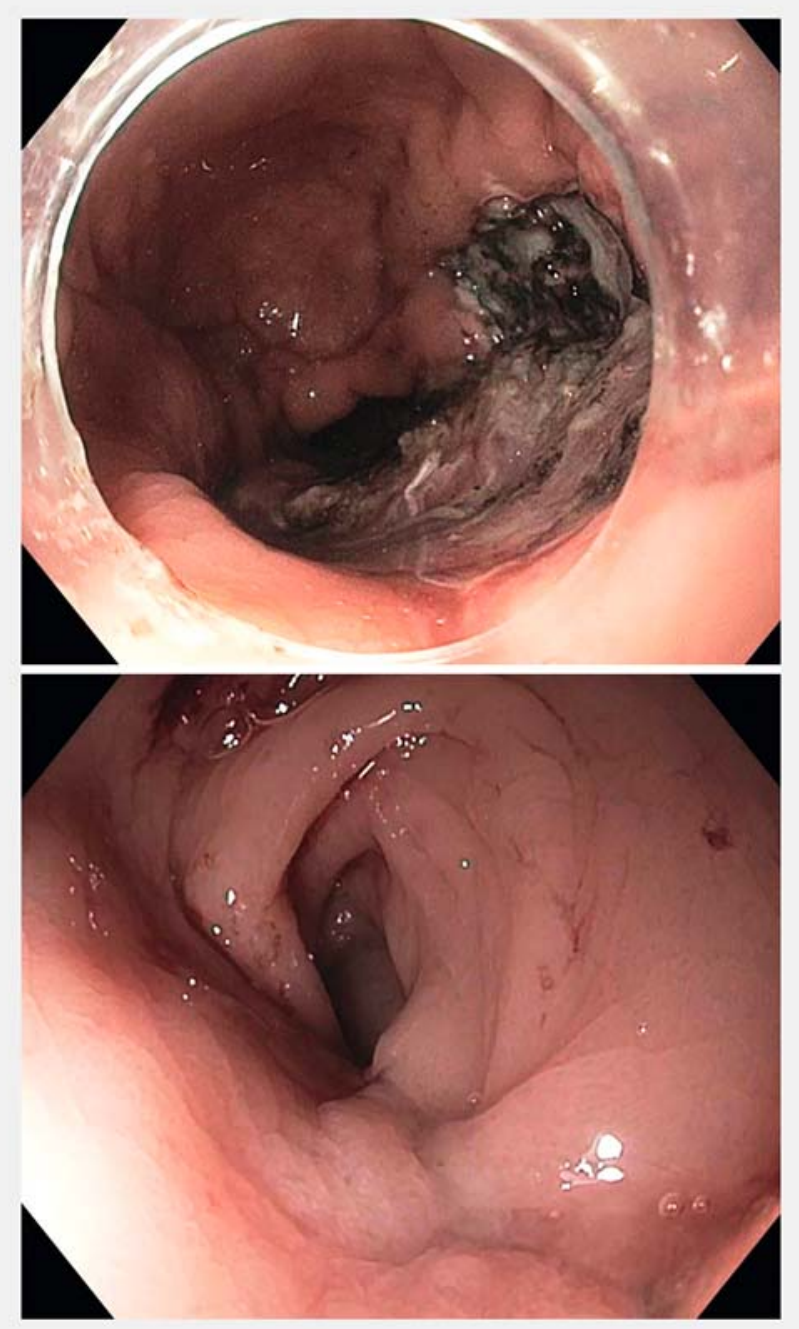

- Fig.4 Rectal endoscopic submucosal dissection defect site before (above) and after (below) endoscopic suturing.

gination into the scar, which could obscure scar evaluation, have been observed, which is likely related to the technique of mucosal/submucosal flap approximation rather than full thickness suturing. Retained suture material ( $\mathbf{F i g . 5}$ ) was found in $19 \%$ of cases and was removed with a loop cutter (Olympus).

\section{Discussion}

In this single-institution prospective study, we demonstrated that endoscopic suturing can be performed safely and effectively to close defects after ESD in the stomach and rectum. Furthermore, endoscopic suturing could be performed efficiently without substantially prolonging procedural times.

The benefit of ESD in providing en bloc resection must be weighed against its significant procedure time, cost, expertise required, and adverse event rate. Delayed bleeding can occur up to 15 days post-procedure and reach rates as high as $4.5 \%$ after gastric ESD $[12,13]$. In patients requiring anticoagulation or antiplatelet agents, minimizing the risk of bleeding is even

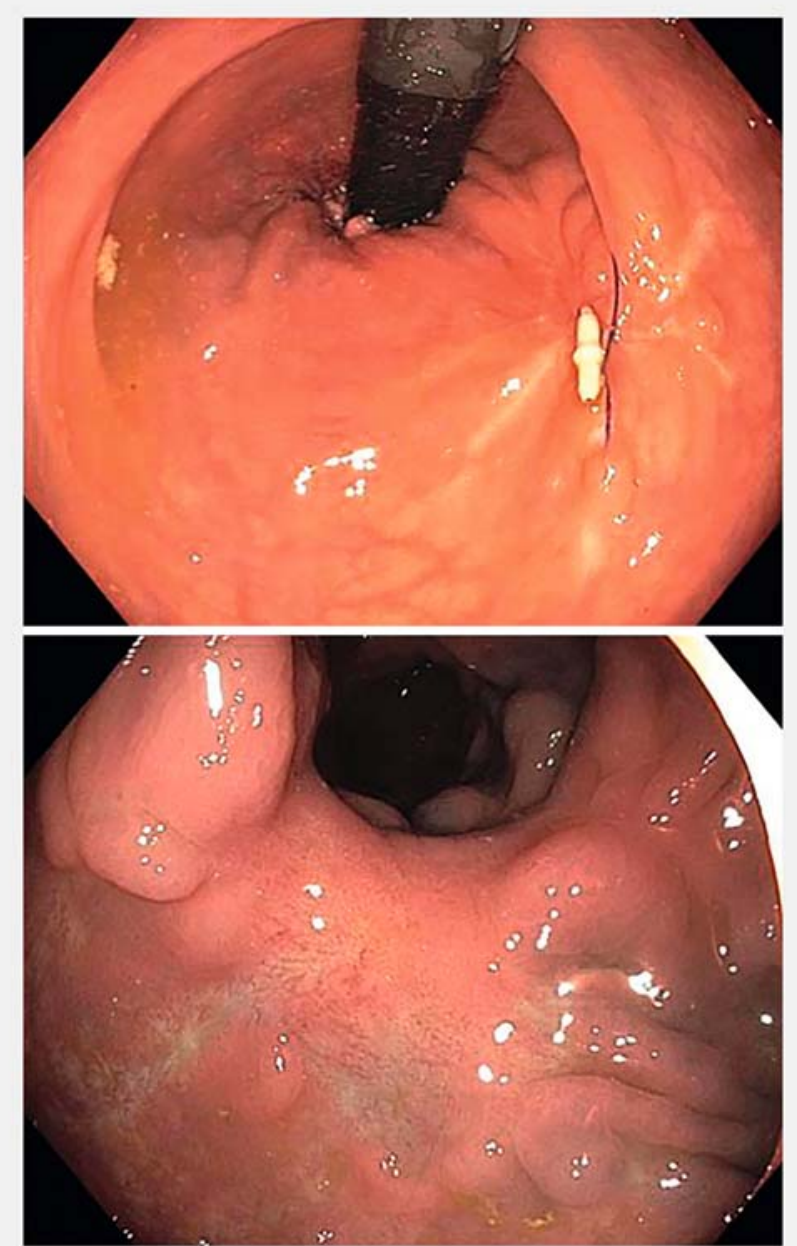

- Fig. 5 Rectal ESD scar with (above) and without (below) retained suture material.

more critical, emphasizing the importance of prophylactic methods. Prior studies examining placement of hemostatic clips have shown rates of bleeding as low as $0 \%$ in the colon, but are challenging to perform in larger defects due to the inability to adequately approximate the defect edges $[3,5,14]$. Furthermore, numerous clips may be required which can add significant time and cost. Endoscopic suturing, on the other hand, was designed to allow for tissue approximation and can be used as salvage therapy in patients who develop bleeding post resection. There were no episodes of delayed bleeding in this study, but larger studies are certainly needed to verify these findings given the relatively low rate of bleeding after ESD.

Perforation represents another feared AE of ESD with rates near $4.5 \%$ after gastric ESD and $4.8 \%$ after colorectal ESD [12, $13,15]$. While most ESD-related perforations occur intra-procedurally and can be immediately treated via clip closure, delayed perforations can occur in a small subset of patients with many requiring emergent surgery [16]. While there were no instances of delayed perforation in our study, the small sample size limits the generalizability of these findings. 
- Table 1 Patient, lesion, and procedure characteristics and patient outcomes.

\begin{tabular}{|c|c|}
\hline Variable & Mean (SD) or $\mathrm{N}(\%)$ \\
\hline Age & $65.6(12.8)$ \\
\hline Male sex & $22(71 \%)$ \\
\hline \multirow[t]{8}{*}{ Lesion location } & Gastric: 18 (58.1\%) \\
\hline & Upper third $(n=8)$ \\
\hline & Middle third $(n=4)$ \\
\hline & Lower third $(n=6)$ \\
\hline & Rectum $13(41.9 \%)$ \\
\hline & Rectosigmoid $(n=3)$ \\
\hline & Upper rectum $(n=6)$ \\
\hline & Lower rectum $(n=4)$ \\
\hline Lesion size $(\mathrm{mm})$ & $27.4(16.2)$ \\
\hline Resected specimen size (mm) & $38.7(16.1)$ \\
\hline \multirow[t]{11}{*}{ Lesion pathology } & Adenocarcinoma: 7 (22.6\%) \\
\hline & Adenoma with high-grade dysplasia: 6 (19.4\%) \\
\hline & Adenoma (low-grade dysplasia): 6 (19.4\%) \\
\hline & Neuroendocrine tumor: 3 (9.7\%) \\
\hline & Lipoma: 2 (6.5\%) \\
\hline & Ulcerated hyperplastic polyp: 2 (6.5\%) \\
\hline & Intestinal metaplasia: 1 (3.2\%) \\
\hline & Gastrointestinal stromal tumor: 1 (3.2\%) \\
\hline & Inflammatory fibroid polyp: 1 (3.2\%) \\
\hline & Fundic gland polyp: 1 (3.2\%) \\
\hline & Spindle cell tumor: 1 (3.2\%) \\
\hline Submucosal dissection time (minutes) & $54.8(34.6)$ \\
\hline Suturing time (minutes) & $13.4(5.9)$ \\
\hline Current antiplatelet/anticoagulation use & $5(16.1 \%)$ \\
\hline Significant intraprocedural bleeding & $2(6.5 \%)$ \\
\hline Intraprocedural perforation & $0(0 \%)$ \\
\hline Same-day discharge & $18(58.1 \%)$ \\
\hline Length of stay if admitted & $1(0.6)$ \\
\hline Delayed bleeding & $0(0 \%)$ \\
\hline Delayed perforation & $0(0 \%)$ \\
\hline Time to surveillance (days) & $143.9(42.2)$ \\
\hline Recurrence & $0(0 \%)$ \\
\hline Retained sutures on surveillance & $4 / 21(19 \%)$ \\
\hline
\end{tabular}

While patients have traditionally been observed in the hospital for several days after ESD, over half the patients in this study could be discharged the same day of the procedure without any AEs. Furthermore, in patients admitted overnight for observa- tion, the vast majority left the following morning, again suggesting that quick discharge can be safe after closure via suturing. Future studies are certainly needed to verify these findings, but the potential cost savings associated with same-day dis- 
charge can be significant and offset the cost of endoscopic suturing in addition to improving patient comfort.

Potential limitations of this endoscopic suturing technique include the prerequisite training and expertise. In addition, this technique requires use of a single-use suturing device along with sutures, cinching devices, and use of a therapeutic gastroscope. Limitations of this study also include sample bias in selecting only gastric and rectal ESD cases as well as its small sample size and lack of a control arm. Furthermore, no cost-effectiveness data were collected to determine the financial implications of endoscopic suturing. From a technical standpoint, lesion size and location can limit the ability to suture the defect. Special attention to suturing lesions involving the esophagogastric junction, pylorus, and anal canal should be made to avoid narrowing of the lumen. While this was not encountered during our study period, massive lesions involving more than half of the lumen's circumference could also be extremely difficult to suture effectively without causing significant narrowing of the lumen.

\section{Conclusion}

In summary, our study describes potential use of endoscopic suturing to close post ESD defect in the stomach and rectum. Further studies are needed to confirm the safety and effectiveness of this technique in preventing AEs associated with ESD. Such studies should include the collection of cost-effectiveness data to compare potential savings of preventing hospital admissions for observation and treatment of AEs with the cost of the suturing devices and procedural time.

\section{Competing interests}

Dr. Wani is a consultant for Boston Scientific and Medtronic. Dr. Edmundowicz is an advisory board member for Olympus. Dr. Soetikno is a consultant for Olympus. Dr. Hammad is a consultant for Medtronic, Olympus, and Cook Medical

\section{References}

[1] Maple JT, Abu Dayyeh BK, Chauhan SS et al. Endoscopic submucosal dissection. Gastrointest Endosc 2015; 81: 1311-1325

[2] Aoki T, Nakajima T, Saito Y et al. Assessment of the validity of the clinical pathway for colon endoscopic submucosal dissection. World journal of gastroenterology 2012; 18: 3721-3726

[3] Ogiyama H, Tsutsui S, Murayama Y et al. Prophylactic clip closure may reduce the risk of delayed bleeding after colorectal endoscopic submucosal dissection. Endosc Int Open 2018; 6: E582-e588

[4] Nomura S, Shimura T, Katano T et al. A multicenter, single-blind randomized controlled trial of endoscopic clipping closure for preventing post-ESD coagulation syndrome. Gastrointest Endosc 2019: doi:10.1016/j.gie.2019.11.030

[5] Harada H, Suehiro S, Murakami D et al. Clinical impact of prophylactic clip closure of mucosal defects after colorectal endoscopic submucosal dissection. Endoscopy international open 2017; 5: E1165e1171

[6] Zhang Y, Wang X, Xiong G et al. Complete defect closure of gastric submucosal tumors with purse-string sutures. Surg Endosc 2014; 28: 1844-1851

[7] Goto O, Oyama T, Ono H et al. Endoscopic hand-suturing is feasible, safe, and might contribute in reducing bleeding risk after gastric endoscopic submucosal dissection: a multicenter pilot study (with video). Gastrointest Endosc 2020: doi:10.1016/j.gie.2019.12.046

[8] Kantsevoy SV, Bitner M, Mitrakov AA et al. Endoscopic suturing closure of large mucosal defects after endoscopic submucosal dissection is technically feasible, fast, and eliminates the need for hospitalization (with videos). Gastrointest Endosc 2014; 79: 503-507

[9] Han S, Wani S, Kaltenbach T et al. Endoscopic suturing for closure of endoscopic submucosal dissection defects. VideoGIE 2019; 4: 310313

[10] Kolb JM, Hammad $H$. The Use of the overstitch to close endoscopic resection defects. Gastrointest Endosc Clin North Am 2020; 30: 163171

[11] Browne RH. On the use of a pilot sample for sample size determination. Stat Med 1995; 14: 1933-1940

[12] Park YM, Cho E, Kang HY et al. The effectiveness and safety of endoscopic submucosal dissection compared with endoscopic mucosal resection for early gastric cancer: a systematic review and metaanalysis. Surg Endosc 2011; 25: 2666-2677

[13] Repici A, Hassan C, De Paula Pessoa D et al. Efficacy and safety of endoscopic submucosal dissection for colorectal neoplasia: a systematic review. Endoscopy 2012; 44: 137-150

[14] Osada T, Sakamoto N, Ritsuno $\mathrm{H}$ et al. Closure with clips to accelerate healing of mucosal defects caused by colorectal endoscopic submucosal dissection. Surg Endosc 2016; 30: 4438-4444

[15] Lian J, Chen S, Zhang Y et al. A meta-analysis of endoscopic submucosal dissection and EMR for early gastric cancer. Gastrointest Endosc 2012; 76: 763-770

[16] Hanaoka N, Uedo N, Ishihara R et al. Clinical features and outcomes of delayed perforation after endoscopic submucosal dissection for early gastric cancer. Endoscopy 2010; 42: 1112-1115 